Pensamiento Crítico Vol. 18 № 1, pp. 143-158

\title{
Evaluación y reflexiones de la inclusión financiera en el perú, a través del mapa estrategico de heimann
}

Ricardo Villamonte Blas

\section{RESUMEN}

La inclusión financiera es uno de los elementos centrales que explican la pobreza. El Mapa Estratégico de Heimann permite obtener un primer diagnóstico transversal de la problemática. En el caso peruano se cuenta con un sistema financiero : bastante sólido, bien supervisado, capitalizado, y líquido, pero se requiere de un plan sistémico que permita reducir gradualmente la exclusión financiera, en razón de que la intervención que realizan los diferentes intermediarios financieros $e$ instituciones involucradas obedecen a enfoques institucionales, no atienden los requerimientos reales de la demanda y no está están articulados. Adicionalmente para que el plan sea sostenible se requiere incorporar estrategias y actividades tanto por el lado de la oferta como de la demanda. El Perú cuenta con instituciones que cuentan con determinada experiencia y con voluntad, se requiere de una convocatoria y un plan de acción dirigido por el Estado.

Palabras clave: Inclusión financiera, mapa estratégico, diagnostico transversal, sistema financiero, plan sistémico, instituciones involucradas, enfoques institucionales, articulación, plan sostenible, Estado. 


\section{Pensamiento Crítico Vol. 18. N I}

\section{SUMMARY}

Financial participation is one of the main components that explain poverty. Heimann Strategic Map allows a first crosscutting assessment of this problem. In Peru there is a financial system which is very solid, well supervised, capitalized, and liquid, but a systematic plan is required to allow gradually reduce financial exclusion, since interventions carried out by different involved financial institutions are due to institutional views. These do not meet real demand requirements and are not organized. In addition to this, in order to make the plan sustainable, it is necessary to incorporate strategies and activities both from supply and demand. Peru has different institutions with certain experience and willingness and it is required an call and an action plan led by the Government.

Keywords: Financial participation, strategicmap, crosscutting assessment (diagnosis), financialsystem, systematicplan, involved institutions, institutional views (or institutional approach), coordination (ororganization), sustainable plan, Government.

\section{INTRODUCCIÓN}

La inclusión financiera es uno de los elementos centrales para la fundamentación de la pobreza, existen en américa latina diferentes enfoques en su conceptualización, como también diferentes diagnósticos y esquemas de trabajo para su atención. El objetivo del presente documento es a partir del Mapa Estratégico de Inclusión Financiera para México elaborado por Ursula Heimann y un equipo de profesionales, realizar un primer diagnóstico para el caso peruano que permitan usar dicho mapa como un esquema central de debate con cargo al cual se podrá elaborar posteriormente un mapa para el país y principalmente un modelo a partir del cual el gobierno y las instituciones involucradas puedan tener una herramienta de trabajo que permita concentrar esfuerzos en procura de implementar políticas y programas tanto del gobierno como de las instituciones públicas y privadas que permitan ir brindando una solución gradual al problema pero de manera integral, y cohesionada.

Es importante señalar que la orientación del presente artículo está referida principalmente a aquellos peruanos que están más excluidos entre ellos podemos referirnos a los que viven en la pobreza y extrema pobreza, que habitan distritos y centros poblados en donde el desarrollo económico es tímido y la presencia del Estado y de las empresas privadas es baja, y se refiere en menor proporción a aquellos peruanos que 


\section{Ricardo Villamonte Blas}

siendo usuarios y más cercanos al sistema financiero requieren de mayores instrumentos para que se les brinde mayores canales y niveles de acceso y profundización financiera.

El mapa estratégico de Ursula Heimann se basa en una adaptación de la metodología del llamado logic framework, o marco lógico, desarrollado en su origen por Leon J. Rosenberg para analizar problemas de forma sistemática y evaluar las opciones para solucionarlos. La estructura del mapeo tiene diferentes dimensiones, bajo las cuales se ordenan varios niveles: los objetivos que se persiguen, las estrategias para alcanzarlos y las actividades necesarias para ponerlas en práctica. Esta metodología es particularmente útil para el estudio de temas y soluciones transversales. Se ha considerado conveniente a fin de ordenar el análisis, resumir los objetivos y estrategias del mapa de Heimann y ubicarlo en recuadros y ,posteriormente hacer una breve evaluación para el caso peruano y proponer actividades pendientes para ir abordando y brindando soluciones a la problemática. Como el amable lector podrá ir evaluando en un artículo académico no se pueden ir abordando todas las soluciones máxime si el problema es transversal a diferentes instituciones público y privadas y en donde se requiere la participación de un equipo multidisciplinario entre ellos: economistas, abogados, administradores , contadores, especialistas en micro finanzas y en pobreza , funcionarios del sistema financiero, funcionarios de los diferentes programas sociales y ministerios como también expertos de la cooperación internacional, por ello el documento pretende motivar a la reflexión de los funcionarios e instituciones involucradas.

El mapa está dividido en dos, la primera parte: la oferta de servicios financieros adecuada a la demanda y, la segunda parte: la demanda consolidada de servicios financieros adecuados y seguros, el cual pasamos a analizar, "vease esquemas 1-A y 1-B"

\section{La oferta de servicios financieros adecuada a la demanda}

\section{Objetivo I : Productos y Servicios Financieros Adecuados al Usuario}

Los productos y servicios financieros que forman parte de la oferta deberán ser adecuados a las necesidades del usuario, es decir, diseñados y operados desde un enfoque de demanda. Dichos servicios requieren responder a necesidades específicas de distintos grupos poblacionales en cuanto a sus características de montos, plazos, costos, requisitos y alcance. 


\section{Pensamiento Crítico Vol. 18. N I}

Para lograr productos y servicios financieros adecuados al usuario, se proponen las siguientes tres estrategias :

1. Impulsar el análisis de las necesidades de la población

2. Incentivar el diseño de productos y servicios financieros adecuados

3. Promover el desarrollo de un entorno competitivo

Si nos referimos a los productos y servicios financieros que requieren los peruanos en situación de pobreza, en el Perú la disponibilidad de ellos parte de un enfoque de oferta institucional que no contempla la problemática a fondo de la demanda . Los diferentes intermediarios financieros involucrados entre ellos : bancos comerciales, COFIDE, cajas municipales, Banco de la Nación, parten de una percepción individual institucional en función de sus niveles de compromiso patrimoniales y de riesgos y a partir de ello diseñan nuevos productos y servicios que no los llevan a pasar significativamente la frontera de sus clientes actuales. Una mayor penetración de financiera deberá analizar la necesidades específicas de distintos grupos poblacionales y sus niveles socio económicos de su capacidad de pago y constitución de garantías. La constitución de garantías requieren un tratamiento especial por cuanto muchas bancos comerciales requerirían de garantías especiales con participación del estado para que los pobres sean sujeto de crédito especialmente los que viven del sector agrícola.

Para la realización de esta tarea se necesita de estudios más profundos sobre las reales necesidades de financiamiento de los excluidos y el diseño de una fina estrategia de intervención. En esta tarea la convocatoria de instituciones y funcionarios involucrados es obligación del Estado como también su compromiso de complementar y coparticipar mediante programas de apoyo relevantes, como por ejemplo de : AGRORURAL y, FONCODES. Estas instituciones cuentan con bajos presupuestos y aun cuando por sus diferentes modelos de intervención son muy conocedoras de los reales requerimientos de financiación de la población pobre con proyectos potenciales emprendedores, no son consideras para la formulación de una estrategia nacional. En el caso de FONCODES recién en los últimos años va dejando su rol inicial de asistencialista y va progresando en sus funciones de apoyar proyectos sociales de emprendimiento familiar ,a través del mejoramiento de capacidades. Uno de sus programas es Mi Chacra Productiva con un diseño y estrategia de intervención muy interesantes pero con recursos financieros 


\section{Ricardo Villamonte Blas}

muy bajos respecto al potencial de demanda que existe. Tanto AGRORURAL como FONCODES pueden aportar muchísimo por cuanto desde hace más de 15 años vienen conociendo y conviviendo con el potencial emprendedor no solo a nivel de los distritos sino a nivel de los centros poblados. Estas instituciones conocen sobre la cultura y organización familiar, sus reales requerimientos de formación, de la problemática de la localidad, cuenca y geografía, de las variantes en la estacionalidad específica de los flujos de ingresos de cada proyecto, de los gastos domésticos de los potenciales clientes y de la disponibilidad de garantías, por lo tanto deben participar en la elaboración de las nuevas estrategias de intervención, pero con una fuerte asignación presupuestal de la república. La participación contundente de estas instituciones permitirá la formación de una base de clientes que se pueden integrar al circuito económico y al sistema financiero nacional. Cuando estos potenciales sujetos de crédito demuestren un buen comportamiento, un cumplimiento de sus obligaciones en la gestión de sus pequeños proyectos podrán incrementar la lista de clientes de las cajas municipales y posteriormente de la banca comercial.

Es importante mencionar la gran experiencia que han obtenido la cooperación internacional y las organizaciones no gubernamentales ( ONGs), sin embargo ese conocimiento se diluye por cuanto manejan proyectos y programas individuales que parten de una focalización individual, y que deben reunirse en un gran proyecto nacional de inclusión financiera.

FONCODES, AGRORUAL han participado en el análisis de las necesidades de la población conjuntamente con el INEI, el MEF, el BCR ( en un primer momento ) tienen experiencia en la elaboración del Mapa de la Pobreza y de herramientas de Focalización, las cuales servirán de base para construir un Mapa de la Riqueza o de las potencialidades emprendedoras a nivel nacional que es el requisito base para establecer una estrategia de intervención integral y sistémica. Este elemento es fundamental por cuanto se lograría, a partir de una sola metodología y estandarización de criterios, establecer la brecha a atender. La brecha debe contemplar los requerimientos de financiación y el diseño de productos y servicios financieros necesarios. Sin ánimo de ser redundante se debe resaltar esta acción por cuanto la labor individual de los diferentes intermediarios viene generando una proliferación de criterios y metodologías de evaluación con un enfoque institucional. 


\section{Pensamiento Crítico Vol. 18. N I}

Logrado ese análisis que permitirá conocer la brecha no atendida, el Estado podrá propiciar la participación de bancos comerciales, bancos públicos, cajas municipales entre otros, y definir el rango de participación de cada uno de ellos y de los programas sociales pero a partir de una presupuesto reforzado que permita atender la financiación de proyectos .

\section{Objetivo II: Productos y servicios financieros con proximidad al usuario}

La razón de la baja inclusión financiera no solamente radica en un problema de productos poco enfocados a satisfacer la demanda de forma masiva. También es un problema de falta de acceso físico a las instituciones que ofrecen servicios financieros formales. La banca comercial generalmente no abre sucursales en ciudades pequeñas o en zonas remotas de un país, lo cual dificulta (si no imposibilita) el acceso a los servicios, además de encarecerlos. Bajo una iniciativa de ampliación de cobertura de los servicios financieros, el tema de la proximidad es esencial y podrá conllevar el incremento en el número de puntos de acceso - sean éstos fijos o móviles- para asegurar cercanía física y conveniencia geográfica a la población objetivo.

Para lograr productos y servicios con proximidad al usuario, se proponen las siguientes tres estrategias :

1. Incentivar el establecimiento de puntos de acceso eficientes

2. Asegurar la sostenibilidad de puntos de acceso

3. Promover la consolidación de los intermediarios financieros

Una enfoque global permite observar que el Perú cuenta con 1,817 distritos en los cuales debería existir la presencia de diferentes canales de atención bancaria que permitan en cada una de ellas brindar los servicios financieros completos que requieran para lograr su desarrollo local e integrarse al circuito económico y social del país. A nivel de las agencias bancarias, quienes son las que brindan la gama completa de servicios de sus entidades, el Banco de la Nación cuenta con 542 agencias y si agregamos a ella la presencia de la banca comercial en su conjunto, las agencias bancarias del sistema financiero atienden en promedio a 800 distritos, lo que permite observar el gran margen de distritos pendientes por atender. El Estado a través del Banco de la Nación 


\section{Ricardo Villamonte Blas}

viene implementando nuevos canales como por ejemplo: la banca móvil ( determinados servicios del banco a través de una unidad móvil por tierra o por ríos con un itinerario determinado y una programación de atención una o dos veces al mes; los agentes bancarios ( presencia bancaria a través de pequeños medios electrónicos para efectuar determinados cobros o retiros de dinero en tiendas o establecimientos comerciales ), ambos se encuentran en una fase de iniciación o de piloto, en este campo la banca comercial todavía no interviene con carácter permanente y no tiene mayor compromiso en atender el gran margen de distritos por atender.

Es importante señalar que el Banco de la Nación diseño un modelo de acercamiento muy interesante y con éxito singular, el cual consistía en permitir que las cajas municipales abrieran una ventanilla de atención en las agencias del banco, gracias a ello las cajas pudieron desarrollar negocios en 270 localidades. Sin embargo el modelo no pudo crecer más por cuanto las agencias del banco son pequeñas y no permiten conceder espacios a las cajas municipales.

Hay varios proyectos pendientes para lograr un mayor acceso, como el proyecto de dinero electrónico ( proyecto del estado ), la banca celular ( proyecto del estado y de la banca comercial ) entre otros. Lo que queda como una tarea pendiente de este documento es el de contabilizar la participación conjunta en cada uno de los distritos y centros poblados de la banca a comercial, banco de la nación y cajas municipales para conocer con objetividad cual es la cantidad de productos y servicios con las que cuenta cada distrito y si estos se ajustan a sus reales, actuales y potenciales requerimientos. Otra tarea pendiente es la de evaluar y proponer acciones concretas para la implementación de las 3 estrategias del presente objetivo.

\section{Objetivo III: Intermediarios financieros consolidados, seguros y estables.}

Un sistema financiero robusto requiere estar conformado por intermediarios financieros consolidados, seguros y estables, capaces de crecer, competir y adaptarse en el tiempo y espacio, además de estar debidamente regulados. Este objetivo está enfocado a garantizar la solidez, seguridad, estabilidad y continuidad de los servicios de los intermediarios financieros a través de consolidar su operación y gobernabilidad, dentro de un marco jurídico adecuado y aplicado de manera decidida. La inclusión financiera debe promoverse a través de intermediarios financieros capaces de realizarla 


\section{Pensamiento Crítico Vol. 18. N I}

con el profesionalismo y solidez necesarios para que ésta sea adecuada, segura, sostenible y permanente, en plena concordancia con la legislación vigente.

Para lograr la consolidación, seguridad y estabilidad de los intermediarios financieros, se proponen las siguientes tres estrategias y sus actividades correspondientes:

1. Promover la consolidación de los intermediarios financieros.

2. Contar con una legislación y regulación adecuadas.

3. Hacer cumplir la legislación.

De las exposiciones de los principales funcionarios de la Superintendencia de Banca, Seguros, y Administradoras Privadas de Fondos de Pensiones (SBS) , y del posterior análisis de gestión y estadístico se puede concluir que el sistema financiero peruano se encuentra en una posición aún más sólida que la que tuvo durante la anterior crisis externa del 2008, lo cual se explica por la acumulación de provisiones y capital social en los últimos años. La banca peruana y el sistema financiero en general están más resguardados, pues en los últimos años han capitalizado el $65 \%$ anual de sus utilidades, inclusive la SBS publicó la regulación de las provisiones procíclicas. El ratio de capital legal es $10 \%$, pero el sistema financiero en promedio llego a operar con un nivel de $14 \%$, lo que implicaba un $40 \%$ de capital adicional por encima de lo requerido legalmente, y en cuanto a las cajas municipales estas han llegado a operar con un ratio de capital más elevado todavía, que se sitúa en $19 \%$ por cuanto son instituciones más pequeñas y más expuestas.

En lo que se refiere al grado de inversión en el Perú, en los últimos años la agencia internacional Standard \& Poor's subió las calificaciones de bancos peruanos entre ellos

el Banco de Crédito del Perú (BCP), el BBVA Banco Continental Scotiabank Perú y COFIDE, los cuales han pasado ahora de BBB- a BBB y mantuvo la calificación de otros dos : Interbank (BBB-/Estable) y de Mibanco (BB+/Estable/B), el Banco de la Nación también ha logrado una aceptable calificación, en general hay una mejor apreciación sobre el sistema financiero peruano.

A entender de los expertos se cuenta con un sistema financiero peruano bastante sólido, bien supervisado, capitalizado, líquido, y aun cuando el Perú es uno de los 


\section{Ricardo Villamonte Blas}

países líderes en microfinanzas, hay segmentos más pequeños, de cajas municipales, donde alguna de ellas deben mejorar su gestión a nivel de prácticas que no fueron apropiadas. Mediante el sistema financiero se puede mejorar en el objetivo de obtener mayor inclusión social, si consideramos que al indicador crédito/PBI, le falta mucho para alcanzar un nivel de intermediación importante, por cuanto estamos a un tercio de lo que se encuentra Chile, sin duda aun pese a la solidez del sistema el Perú tiene mucho margen para mejorar.

\section{La demanda consolidada de servicios financieros adecuados y seguros}

\section{Objetivo I: Protección y defensa del usuario}

La confianza es un ingrediente crucial de la inclusión financiera. Para ello es necesario que se construyan instituciones y mecanismos de protección y defensa cuya efectividad y funcionamiento sean ampliamente conocidos por los usuarios.

Para lograr la protección y defensa del usuario se proponen las siguientes dos estrategias :

1. Contar con instituciones y mecanismos efectivos de protección y defensa del usuario.

2. Impulsar la transparencia de los intermediarios financieros.

El Perú cuenta con un marco normativo de la Superintendencia de Banca y Seguros a través de la Resolución No 818 -2012 que se refiere al Reglamento de Transparencia de Información y Contratación con Usuarios del Sistema Financiero , el cual considerada entre otros temas: la transparencia de información sobre operaciones activas y pasivas; criterios para la determinación de tasas de interés, comisiones, gastos y rendimientos con mecanismos de difusión; información que debe ser entregada al usuario para efectos de contratación y prestación de operaciones activas, pasivas y de servicios; modificaciones de tasas, comisiones y gastos; difusión y supervisión de fórmulas y programas para la liquidación de intereses y pagos; clausulas generales de contratación ; cláusulas abusivas; información periódica que las empresas deben alcanzar al usuario; implementación de un sistema de atención al usuario, integrando las normas precedentes de observancia obligatoria del INDECOPI; y responsabilidades del 


\section{Pensamiento Crítico Vol. 18. N I}

oficial de atención al usuario. Asimismo el INDECOPI mediante Ley 29571 implemento el Código de Protección y Defensa del Consumidor, considera en ella la protección al consumidor de productos o servicios financieros y brinda el marco normativo de responsabilidades y sanciones .

Finalmente el Perú cuenta con la Ley No 27806 de Transparencia y Acceso a la Información Pública y mediante el FONAFE se obliga a los intermediarios financieros del Estado a brindar al público en general información fidedigna, oportuna sobre los productos y servicios que ofrecen, su situación financiera, sus políticas entre otros.

Lo que debe hacerse en adelante es velar por la adecuada instrumentalización por parte de todos los miembros del sistema financiero para asegurarse que los mecanismos de protección al usuario funcionen permanentemente de manera eficiente y efectiva y , asegurar una adecuada difusión para que los mecanismos de protección y defensa de los usuarios sean cada vez más útiles.

\section{Objetivo II: Comprensión de y confianza en los intermediarios financieros y sus productos y servicios}

Con este objetivo se pretende que los usuarios entiendan de manera general el sistema financiero de su país y conozcan las funciones y características de los intermediarios financieros que lo integran, además de su oferta de productos y servicios financieros. Esto incluye estar informados de manera general sobre los riesgos, costos y ventajas comparativas entre productos y servicios, a fin de permitir una selección de los mismos de manera informada, óptima y prudente.

Para lograr una comprensión óptima de los servicios y productos por parte de los usuarios, se plantean dos estrategias :

1. Fortalecer el entendimiento general del sistema financiero y los intermediarios financieros.

2. Educar sobre el uso adecuado de los productos y servicios financieros. 


\section{Ricardo Villamonte Blas}

La Superintendencia de Banca , Seguros y AFP ( SBS )realiza entre otras las siguientes actividades en materia de Educación Financiera :la Campaña de la Cultura Financiera, la cual tiene como finalidad difundir entre la población, contenidos que le permitan tomar un rol activo en el manejo de sus finanzas personales y familiares, dándoles las herramientas necesarias para tener un mejor desempeño al utilizar los productos y servicios de los sistemas financieros, así como desarrollar habilidades para el buen uso y manejo de su dinero; charlas de cultura financiera para empresas, las charlas están dirigidas a empresas, entidades $e$ instituciones públicas o privadas que deseen capacitar gratuitamente a su personal sobre el buen manejo de las finanzas personales, un portal de educación financiera con consejos para las finanzas personales: manejo de tarjeta de crédito, elección de un crédito , ahorro; un Programa de Asesoría a Docentes : con la finalidad de promover la inclusión financiera la SBS suscribió un Convenio de Cooperación con el Ministerio de Educación en junio de 2006.

En general, de su portal de educación financiera se denota una preocupación de la SBS que debe profundizarse aún más en su programaciones y ampliar más sus contenidos y luego propiciar un despliegue a nivel nacional a través de los diversos intermediarios financieros. Asimismo hay algunas instituciones de la cooperación internacional pública y privada , como las de Alemania que tienen experiencias y programas de educación financiera ya validados en otros países y que intentan difundirlos a través del Banco de la Nación y de las Cajas Municipales.

De la revisión efectuada de los intermediarios financieros nos encontramos con el hecho de que hay diferentes programas, con diferentes planes de estudio, sílabos y cronogramas, que si bien denotan una presencia y preocupación por la educación financiera esta no está articulada, y esta diferenciadamente constituida. Se requiere de un diagnostico global por parte del Estado que no solo estandarice los requerimientos sino su aplicación en un marco de intervención conjunta con los diferentes intermediarios financieros.

\section{Objetivo III: Comportamientos financieros personales responsables}

Con este objetivo se persigue que el usuario logre una planeación y mejor uso de sus recursos, que adquiera habilidades para la toma de decisiones económicas y 


\section{Pensamiento Crítico Vol. 18. No I}

financieras, así como que comprenda, de manera general, el papel que juega en el sistema económico y financiero.

\section{Estrategias:}

1. Educar sobre el uso adecuado de los productos y servicios financieros.

2. Implementar estrategias estructuradas de educación financiera.

La Educación Financiera es un término que ha venido tomando mayor relevancia en los últimos años, como consecuencia de la necesidad de saber cómo mejorar la situación financiera actual y futura de las personas y de las familias.

Para la Organización para la Cooperación y el Desarrollo Económico, la Educación Financiera, es el proceso mediante el cual, tanto los consumidores como los inversionistas

financieros logran un mejor conocimiento de los diferentes productos financieros, de sus riesgos y de sus beneficios, y que mediante la información o instrucción, puedan desarrollar habilidades que les permiten una mejor toma de decisiones, lo que deriva en un mayor bienestar económico. La educación financiera permite el acceso de las personas a información y herramientas sobre el funcionamiento de la economía y las finanzas; incide y es importante en la vida diaria, otorgando la confianza que da el conocimiento, en la toma de decisiones. A la educación financiera se le viene concibiendo como parte del proceso de socialización que va en dos direcciones : quien la proporciona y quien la recibe y, que debe darse a lo largo de toda la vida, por lo que es continuo $e$ inherente al ser humano.

La relevancia es mayor si apreciamos las necesidades que hay en las zonas rurales en las cuales se requiere primero de una alfabetización financiera, que se refiere en el caso de niños y principalmente pobres a la preparación necesaria para desarrollar la habilidad de realizar juicios informados y tomar decisiones efectivas respecto al uso y manejo del dinero.

A nivel de los excluidos que están ubicados en zonas y centros poblados en donde no hay una presencia de la banca comercial y en donde los bancos públicos van lentamente iniciando su presencia, se conoce que en los diferentes modelos de intervención de varias 


\section{Ricardo Villamonte Blas}

ONG's, Organismos de Cooperación Internacional, Programas Sociales, se establecen algunos componentes vinculados a la alfabetización y educación financiera a fin de que los excluidos adquieran habilidades para la toma de decisiones económicas familiares y/o empresariales y para que obtengan un nivel de conocimiento muy genérico sobre el papel que juega el sistema financiero. Mención especial debe hacerse sobre la actuación de algunas caja municipales y ONG's como por ejemplo el caso de CARE en Ayacucho , en donde se ejecuta un proyecto que consiste en capacitar a las mujeres que forman parte del programa JUNTOS para facilitar su acceso al sistema financiero mediante el ahorro, los microcréditos y préstamos, con la finalidad de promover el desarrollo de sus familias para superar las condiciones de pobreza extrema en las que viven. En general debe reconocerse que no parten de un programa integral especifico orientados al uso adecuado de productos y servicios y a la integración financiera del excluido . Los logros que se han ido viabilizando son pequeños por cuanto no parten de un programa integral declarado de interés nacional con criterios estandarizados y que hayan provenido de un estudio que considere objetivos y estrategias nacionales, en este campo hay mucho trabajo por realizar.

\section{CONCLUSIONES}

El mapa de Heimann es interesante y útil porque permite:

- Abordar sistemáticamente el tema de la exclusión financiera el cual debe ser transversalmente tratado por la cantidad de instituciones involucradas.

- Hacer más notorio que la actuación actual de las entidades involucradas esta desarticulada y no tiene una concepción integral como tampoco sistémica.

- Apreciar que por su experiencia : los programas sociales, organismos internacionales cooperantes, ONG's y cajas municipales en el apoyo de proyectos sociales de emprendimiento son muy importantes y se constituyen en los agentes básicos de la inclusión financiera en el Perú.

- Observar que la participación del Banco de la Nación, es importante en la procura del desarrollo de mayor acceso pero, que debe ser complementado 


\section{Pensamiento Crítico Vol. 18. No I}

por el conjunto de intermediarios financieros para lograr un mayor acceso y profundidad en los productos y servicios financieros que requieren los excluidos.

- Destacar que el tema de la educación y alfabetización financiera es importante no solo para asegurar el servicio de las entidades financieras sino para el desarrollo y la integración social de los pobres .

- Apreciar que el Perú cuenta con un sistema financiero sólido y bien supervisado pero que requiere ser reorientado para la consecución de un una política financiera social que permita profundizar y dar un mayor acceso a la inclusión financiera principalmente a nivel de los distritos no atendidos.

- Destacar que el Estado debe convocar a todas las entidades involucradas para la constitución de un plan sistémico de inclusión financiera.

\section{BIBLIOGRAFÍA}

BANCO DE INDONESIA. Informe sobre el Foro Mundial de Políticas de Inclusión Financiera de AFI 2010; Alliance for Financial Inclusion, Indonesia 2010

CHODWHURY, NAZRUL I ; PEREZ SANCHEZ , CARMEN. "Microfinanzas para la inclusión social y financiera: modelos para asegurar que nadie sea excluido del acceso a oportunidades" ; Cumbre Mundial del Microcrédito 2011 , 14 al 17 de noviembre del 2011 - Valladolid, España

DIARIO LA PRIMERA- PERU. "Sistema financiero peruano es seguro", Jueves 26 de enero del 2012

EL COMERCIO-PERU. "Standard \& Poor's mejoró la calificación crediticia de cuatro bancos peruanos", Martes 6 de setiembre del 2011.

HEIMANN, URSULA; NAVARRETE, JUAN; O’KEFEE, MARIA; VACA, BEATRIZ; ZAPATA ,GABRIELA "Mapa Estratégico de Inclusión Financiera: Una herramienta de Análisis; Inclusión Financiera", Sparkassenstiftung für Internationale Kooperation, Ciudad de México, 2009 


\section{Ricardo Villamonte Blas}

MARTINEZ ROLLAND, MIGUEL ANGEL. "II Jornada de Inclusión y Desarrollo: regulación, innovación y educación- Los trabajos del G-20 sobre inclusión financiera"; Dirección General de Financiación Internacional; Ministerio de Economía y Hacienda, 26 de octubre del 2010.

PODER LEGISLATIVO - PRESIDENCIA DEL CONSEJO DE MINISTROS. Ley 29571, Código de Protección y Defensa del Consumidor

PODER LEGISLATIVO - PRESIDENCIA DEL CONSEJO DE MINISTROS. Ley N 27806 , Transparencia y Acceso a la Información Pública

SUPERINTENDENCIA DE BANCA, SEGUROS Y ADMINISTRADORAS PRIVADAS DE FONDOS DE PENSIONES. Resolución N 818-2012, Reglamento de Transparencia de Información y Contratación con Usuarios del Sistema Financiero 


\section{Pensamiento Crítico Vol. I8. $\mathrm{N}^{\circ}$ I}

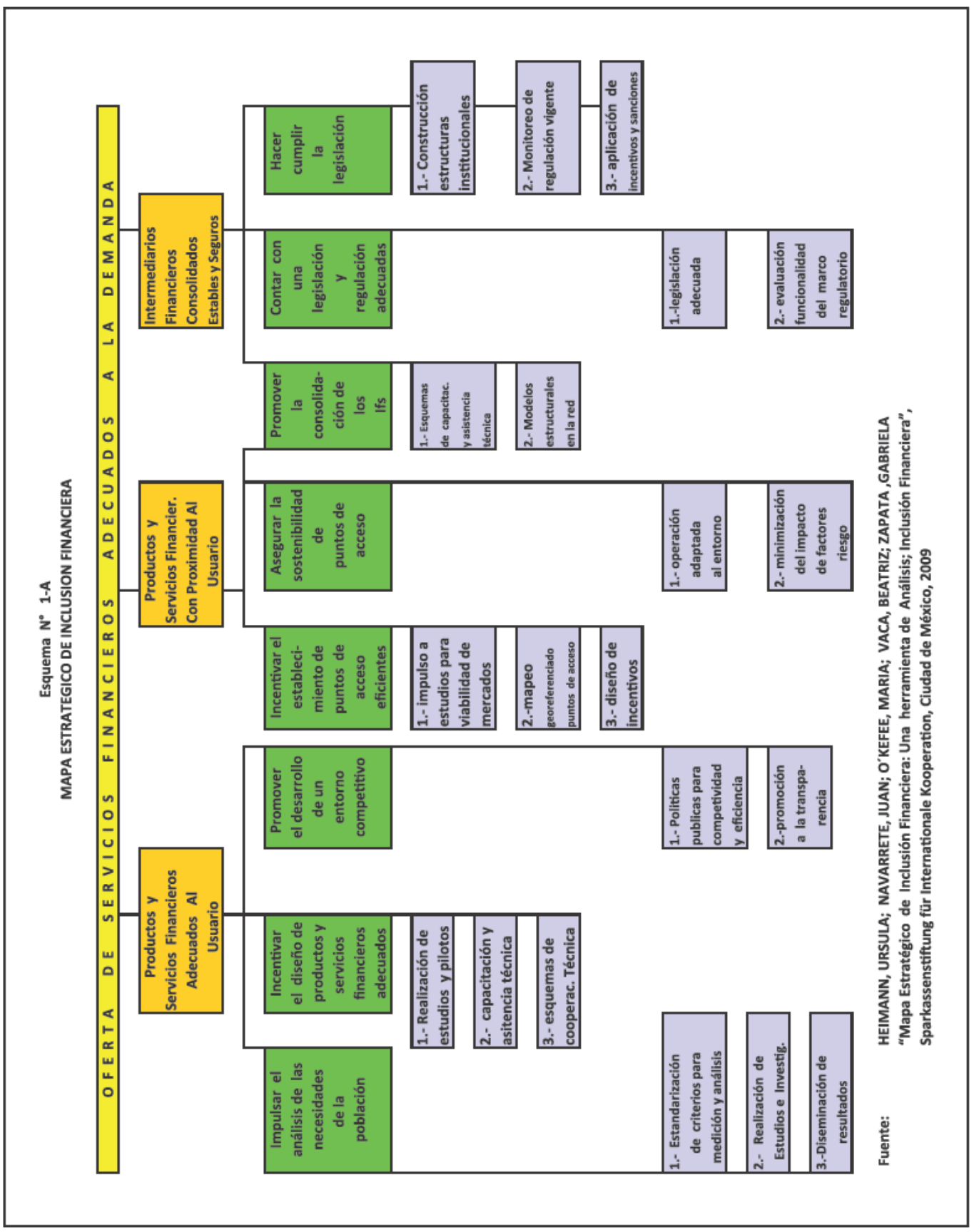

